Projets

de paysage

\section{Projets de paysage}

Revue scientifique sur la conception et l'aménagement de l'espace

$17 \mid 2017$

Paysage(s) et agriculture(s)

\title{
De la mise en valeur du patrimoine viticole à l'émergence d'un paysage culturel au Chili
}

De la puesta en valor del patrimonio vitícola al surgimiento de un paisaje cultural en Chile

\section{Claudia Márquez Thomas}

\section{(2) OpenEdition}

\section{Journals}

Édition électronique

URL : http://journals.openedition.org/paysage/4736

DOI : $10.4000 /$ paysage.4736

ISSN : 1969-6124

Éditeur :

École nationale supérieure du paysage de Versailles-Marseille, Institut national des sciences appliquées Centre Val de Loire - École de la nature et du paysage, École nationale supérieure d'architecture et de paysage de Bordeaux, École nationale supérieure d'architecture et de paysage de Lille, Agrocampus Angers

\section{Référence électronique}

Claudia Márquez Thomas, « De la mise en valeur du patrimoine viticole à l'émergence d'un paysage culturel au Chili », Projets de paysage [En ligne], 17 | 2017, mis en ligne le, consulté le 03 avril 2020 URL : http://journals.openedition.org/paysage/4736 ; DOI : https://doi.org/10.4000/paysage.4736

Ce document a été généré automatiquement le 3 avril 2020.

Projets de paysage 


\title{
De la mise en valeur du patrimoine viticole à l'émergence d'un paysage culturel au Chili
}

\author{
De la puesta en valor del patrimonio vitícola al surgimiento de un paisaje \\ cultural en Chile
}

Claudia Márquez Thomas

Dans un contexte mondial où la viticulture est alliée au tourisme, et ceci d'autant plus dans le nouveau monde viticole de l'Amérique latine, le tourisme est censé faciliter la commercialisation et le marketing de l'activité agricole, tandis que la viticulture permettrait d'enrichir à son tour l'offre touristique locale, de développer l'économie de ces régions rurales, tout en valorisant leurs paysages viticoles, dont la qualité serait un signe de l'excellence des vins, au même titre que le savoir-faire des producteurs (Lignon-Darmaillac, 2014).

2 Pourtant, dans des pays à tradition viticole importante, comme le Chili, l'émergence et la mise en valeur des paysages culturels viticoles n'ont débuté qu'à une date récente (dernière décennie), alors que la législation consacrée aux paysages naturels du pays existe depuis le début du siècle dernier. Ce phénomène résulte des efforts entrepris par les producteurs et les organismes publics, en résonance avec le secteur universitaire, à travers des stratégies de marketing dans le but de mettre en avant l'image d'une production traditionnelle à valeur patrimoniale et pour positionner l'œnotourisme qui lui serait associé. Nous nous questionnons dans cet article sur l'apparition de la catégorie de paysage culturel, alors que celle-ci n'existe pas dans le cadre législatif chilien : de quelle façon a-t-elle émergé dans le contexte viticole chilien? Comment et par qui ce paysage culturel viticole est-il pris en compte dans ce processus?

Nous retraçons ici l'évolution vers la prise en compte et la mise en protection du paysage culturel agricole sur la scène chilienne (première partie), pour ensuite décrire le contexte de l'activité viticole des dernières décennies qui a permis le début d'une reconnaissance (deuxième partie); enfin, nous analyserons le processus menant à 
l'apparition de cette catégorie en rapport avec une démarche de mise en valeur patrimoniale et des stratégies commerciales et œnotouristiques de la viticulture nationale, ainsi que leur prise en compte par les acteurs concernés (troisième partie).

\section{Le contexte de protection et de patrimonialisation des paysages culturels au Chili}

4 Le Chili ne possède encore aucune loi de paysage en tant que tel. Certes, l'on peut considérer que la notion de protection de paysage dans la législation chilienne existe dès la première moitié du $\mathrm{xx}^{\mathrm{e}}$ siècle, avec la création du premier parc national du pays (en 1926), lequel visait la conservation des ressources naturelles et des «beautés scéniques" (bellezas escénicas). L'objectif prioritaire était alors la promotion du tourisme, auquel se subordonnait celui de la protection des paysages (Corporación Nacional Forestal [CONAF], 2015). Par ailleurs, divers textes de loi régulant l'usage des ressources naturelles évoquent alors la protection de la beauté des paysages (loi nationale des forêts de 1925 par exemple), des panoramas ou paysages extraordinaires (Convention de Washington de l'OEA de 1940, ratifiée par le Chili en 1967). Cependant, ces textes ne font allusion qu'aux paysages naturels - le plus souvent - remarquables. Cette idée est renforcée par la suite grâce à la Convention sur la diversité biologique (Rio, 1992), ratifiée par le Chili en 1994, qui prend en considération, entre autres, des valeurs esthétiques de la diversité biologique et ses composantes.

Ce lien entre conservation des éléments naturels, maintien de la " qualité paysagère " et tourisme fut renforcé ensuite par la loi de protection environnementale du Chili de 1994 (loi n 19300 de Bases générales de l'environnement, modifiée en 2010 par la loi $n^{\circ} 20$ 417) - la seule à faire allusion au paysage. Cette dernière, qui établit entre autres les normes d'évaluation de l'impact des projets d'investissement sur l'environnement, intègre le paysage comme ressource naturelle et composante environnementale. Mis sous protection, il est à considérer donc lorsque ces projets peuvent altérer de façon significative la valeur paysagère ou touristique des zones où ils seront situés. En complément, le règlement de cette loi (de 1997, modifié en 2012) définit qu'une zone possède une valeur paysagère "lorsque, étant visuellement perceptible, elle possède des attributs naturels qui lui confèrent une qualité qui la rend unique et représentative " (article $n^{\circ} 2$, titre $I$, décret suprême $n^{\circ} 40$ de 2012, ministère de l'Environnement $^{1}$ ). Si la définition précise du terme « paysage » n'est pas donnée dans ce texte de loi ni dans son règlement, c'est sur ses composantes naturelles et leur qualité « unique » et « représentative » que l'accent est mis.

Une valorisation esthétique des paysages naturels possédant un potentiel touristique existe ainsi de longue date dans la législation environnementale du pays. Toutefois, à ce jour, elle ne tient pas compte des paysages à composantes anthropiques. Ainsi, la présence de terrains privés productifs à l'intérieur des espaces protégés a souvent posé problème en raison de l'opposition de leurs objectifs. Les paysages culturels des zones «productives » ont eu du mal aussi à trouver leur place - pourtant croissante - dans la législation en matière de patrimoine culturel, qui débute dans le pays en 1925 avec la création du Conseil de monuments nationaux (CMN).

7 La loi Bases générales de l'environnement citée précédemment, par exemple, souligne l'importance des composantes du patrimoine culturel (sites à valeur archéologique, 
historique), sans faire référence aux paysages culturels ; mais elle se rapproche de cette notion en indiquant dans son règlement que les études d'impact doivent contenir la description « des éléments naturels et artificiels » qui composent ce patrimoine (article $\mathrm{n}^{\circ} 18$, titre III, ministère de l'Environnement). Plus ancienne, la loi sur les monuments nationaux de 1970 (loi n 17 288) renvoie à une figure de protection proche de l'idée de paysage culturel et même agraire : zones typiques ou pittoresques. Ces dernières, ayant connu une évolution depuis la loi de 1970, sont définies par la norme du CMN de 2001 comme: "des ensembles d'immeubles urbains ou ruraux qui forment une unité d'établissement représentatif de l'évolution d'une communauté humaine et qui se démarquent par leur unité stylistique, leur matérialité ou leurs techniques de construction; [...] constituant des aires liées par les bâtiments et le paysage qui les encadre, les souligne et les met en relation, pour former une unité paysagère, à caractéristiques environnementales propres, qui les définissent et qui leur donnent une identité, une référence historique et urbaine dans un lieu, village ou ville ». Avec ces normes, auxquelles s'ajoute le récent règlement (Ministerio de Educación, 2017), la prise en considération du paysage dans ces zones est affirmée : celles-ci doivent être à l'abri d'interventions impliquant des discordances visuelles avec leurs caractéristiques architecturales, urbaines mais aussi paysagères, leurs espaces naturels devant être également protégés. Il existe quatre catégories de zones typiques ou pittoresques: village traditionnel, centre historique, alentours de monument historique, zone et ensemble urbains. On compte actuellement seulement cinq zones à composantes essentiellement rurales (la première date de 1974, la dernière de 2008), principalement des haciendas de quelques hectares, dénotant une sensibilité aux expressions territoriales attachées à certains éléments du patrimoine naturel et culturel en contexte agricole.

8 D'autre part, le Chili a ratifié en 1980 la Convention du patrimoine mondial de l'Unesco de 1972, et compte six sites inscrits au patrimoine mondial, le premier proclamé en 1995. Or, parmi ceux-ci, aucun ne figure dans la catégorie de paysage culturel établie par la Convention en 1992 (premier instrument juridique international en la matière), même si certains d'entre eux correspondent à la définition donnée («ouvrages combinés de la nature et de l'homme », Unesco, 1992).

Quant aux instruments de planification territoriale, entre les milieux urbains et les espaces naturels protégés, les paysages culturels productifs semblent être encore une catégorie difficile à cerner. Ainsi, les zones agricoles ne bénéficient pas à ce jour de régulations en matière de paysage et sont dépendantes des normes d'occupation des sols établies par des plans directeurs urbains, communaux et intercommunaux. Cependant, les aires urbaines se développent souvent sans prendre en compte leurs liens avec les aires rurales (Miranda et al., 2015), lesquelles restent souvent vulnérables face aux autorisations de changement d'utilisation du sol favorisant l'expansion urbaine (Oficina de estudios y políticas agrarias [ODEPA], 2013). De plus, le plan national pour le développement rural de 2014 énonce des objectifs pour le développement durable de ces secteurs, en parallèle avec leur valorisation culturelle, identitaire et patrimoniale - encore peu explorée -, et la promotion d'un aménagement du territoire adapté au maintien du paysage (Ministerio de Agricultura, 2014). À remarquer toutefois: le paysage est ici cité dans l'axe stratégique de «biodiversité, services écosystémiques et paysage", ayant pour objectif de "promouvoir l'identification des paysages naturels et semi-naturels du Chili, reconnaissant et intégrant le paysage dans les politiques sectorielles» (Ministerio de Agricultura, 2014). 
Même dans le contexte d'une politique agricole, le paysage culturel semble ici assimilé au concept de " semi-naturel », juste après les paysages naturels.

Ainsi, certains paysages naturels du pays ont été depuis des décennies nommés comme tels et mis sous protection, tandis que les paysages culturels - agraires ou autres - n'ont pas été intégrés en ces termes dans le cadre de protection. S'ils sont protégés en tant que zone typique ou pour coïncider avec des lieux à haute valeur naturelle, par exemple, ils ne sont pas reconnus comme tels. Cette "non-reconnaissance» est comparable à la situation connue dans d'autres pays européens au début des années 2000, comme en Espagne, lorsque la conservation du paysage était associée à la politique d'espaces naturels protégés, malgré la dimension culturelle de la plupart d'entre eux ${ }^{2}$. Pourtant, la loi espagnole 4/1989 de conservation des espaces naturels et de la flore et faune sauvages en vigueur jusqu'en 2007 établissait le principe de " paysage protégé ", laquelle, tout en faisant référence à des emplacements spécifiques du milieu naturel, pouvait comprendre - en particulier dans les normes régionales des systèmes agraires à intérêt écologique ou culturel (Mata Olmo, 2004).

À ce jour, le manque d'articulation des normes pour une meilleure prise en compte du paysage est manifeste dans les différents secteurs liés à l'aménagement du territoire chilien (Miranda et al., 2015). De même, l'absence d'une vision qui regrouperait les valeurs naturelles et culturelles du territoire, sous une définition intégrale du paysage qui conceptualise et protège son usage - comme le fait la Convention européenne du paysage - représente un défi certain pour la reconnaissance des paysages culturels de toute l'Amérique latine (Instituto de la Patagonia, Icomos et Universidad de Magallanes, 2013).

Ce paradigme de protection des paysages a pourtant commencé à changer dans le pays ces quinze dernières années. On le doit en partie à un intérêt académique croissant pour les paysages et les paysages culturels en particulier, de la part d'universités, d'organismes gouvernementaux et d'associations latino-américaines qui se consacrent à la thématique ${ }^{3}$, comme en écho au courant mondial - et européen notamment - de la protection de ces paysages. Ces dernières mettent l'accent sur la nécessité d'une législation dans le domaine et de la mise en œuvre d'outils de gestion comme les catalogues et chartes de paysage. Il est à noter également que ce changement se produit au fur et à mesure que la valorisation de certaines agricultures locales et de leurs paysages s'impose dans le pays et la région, tout en visant leur développement touristique et/ou durable ${ }^{4}$.

13 Pourtant, malgré l'inexistence d'un cadre conceptuel et législatif, la notion de paysage culturel est apparue suite à l'évolution de la valorisation du patrimoine viticole et de l'image commerciale de la production viticole nationale, accompagnée du développement de l'œnotourisme. Après une mise en contexte de la viticulture chilienne où cette émergence s'est produite, nous exposerons le processus qui a mené à cette prise en compte de la viticulture en tant que paysage culturel dans le pays. 


\section{La viticulture chilienne et l'essor de l'œnotourisme ces vingt dernières années comme condition propice à la reconnaissance des paysages}

14 Le Chili possède une longue et importante tradition vitivinicole. Celle-ci remonte à l'époque des conquistadors pour qui, dès leur arrivée (vers 1540-1550) dans la vallée centrale du pays et les premières décennies qui suivront, le vin est un élément de la vie domestique et eucharistique de la colonisation hispanique (Del Pozo, 1999 ; Nahrwold, 2014 ; Rojas, 2015). Avec une production permettant l'exportation des excédents dès le $\mathrm{xVI}^{\mathrm{e}}$ siècle, la viticulture chilienne aurait généré au cours du $\mathrm{xVIII}^{\mathrm{e}}$ siècle un début d'industrie locale. Puis, au xix siècle, avec une expansion économique et productive importante ainsi que la modernisation de la viticulture par l'introduction de nouvelles techniques agricoles d'influence française (l'introduction de cépages européens, par exemple ${ }^{5}$ ), le Chili commence à exporter du vin vers l'Europe en 1877. Il compte déjà en 1883 sur la scène internationale, avec une douzaine de "grands " établissements vinicoles exportateurs dans le centre du pays et environ 5000 hectares plantés de vignes en 1897 (Rojas, 2015). Après une période de déclin au xxe siècle, c'est au cours des années 1980 que l'industrie viticole connaît une croissance forte, avec l'augmentation des exportations (Lacoste, 2005) et l'apparition de nouveaux vignobles financés souvent par des investissements étrangers. Elle progresse encore dans les deux dernières décennies, avec l'exploration de nouveaux territoires viticoles et une importante amélioration de la qualité des vins (Del Pozo, 1999 ; Nahrwold, 2014 ; Rojas, 2015). Ce développement important de la culture se traduit spatialement par une augmentation des surfaces cultivées dans le pays, principalement celles qui sont irriguées ${ }^{6}$, les vignes montant souvent le long des versants des vallées et devenant de plus en plus un vignoble de piémont (Faliet, 2005).

Grâce à un investissement technologique considérable dans l'industrie ces dernières années (Zamora et Barril, 2007) et d'efforts conséquents pour améliorer l'image du vin chilien sur le marché intérieur comme à l'étranger (Rojas, 2015), le pays présente aujourd'hui une importante production viticole, à fort dynamisme, le situant en 2016 au quatrième rang des exportateurs dans le monde (le Chili exporte la plus grande partie de sa production vers 150 pays), et au sixième rang des producteurs ${ }^{7}$ (soit $8 \%$ de la production mondiale). Il apparaît comme l'un des principaux acteurs des pays du nouveau monde viticole (Observatoire espagnol du marché du vin cité par Infyde, 2016). Ce rôle déterminant d'exportateur, consolidé au début des années 1990 (Del Pozo, 1999), a contribué à la création des dénominations d'origine (DO) des vignobles chiliens en 1985 (loi n 18455 et modifications postérieures), ainsi qu'à l'établissement $\mathrm{du}$ concept de terroir, repris textuellement du français, afin de lier producteurs et consommateurs et de distinguer les vins propres à chaque région.

En parallèle à cette augmentation d'une production viticole de plus en plus renommée internationalement et à l'augmentation du tourisme dans le pays ces dernières années ${ }^{8}$, l'œnotourisme, en tant que tourisme spécialisé réunissant l'industrie du tourisme et l'industrie du vin, bénéficie d'une croissance importante de visiteurs avec une moyenne annuelle de $10 \%$ au cours des dix dernières années (Godoy et Meza, 2014 ; Infyde, 2016).

Vues et conçues comme un moyen d'augmenter les ventes (surtout dans les périodes critiques), les premières initiatives d'ouverture des caves au public ont débuté au Chili 
dans les années 1990 (vignoble Miguel Torres dans la Vallée de Curicó); et c'est en 1996 qu'est aménagée la première route du vin dans la vallée de Colchagua. Toutefois, malgré les investissements tant publics que privés, les débuts de ces routes et de quelques autres initiatives ont été laborieux, et ce n'est que dans les années 2000 que leurs activités ont réellement repris, non sans difficulté, en se concentrant sur les visiteurs de vignobles (Zamora et Barril, 2007), dans un contexte mondial où l'œnotourisme serait devenu un élément incontournable du marketing des sociétés viticoles (Lignon-Darmaillac, 2014). Le déploiement de ce nouveau moyen de fidélisation des consommateurs dans le pays est donc récent par rapport au développement de la production viticole des dernières décennies (Inalaf et al., 2012), mais aussi en comparaison avec d'autres nouveaux pays viticoles. Cet essor récent de l'œnotourisme national s'est nourri de différents projets mis en place par les secteurs public (service national du Tourisme [Sernatur] et sous-secrétariat au Tourisme) et privé (association professionnelle Vinos de Chile - créée en 2007 -, Federación de Empresas de Turismo de Chile [Fedetur], principalement), afin de diversifier l'offre touristique du pays et d'élargir les segments de la demande nationale et internationale ${ }^{9}$.

L'œnotourisme au Chili est étroitement lié aux vallées viticoles et à leur localisation : constitués de cinq régions viticoles regroupant dix-neuf vallées du nord au sud du territoire (de $29^{\circ}$ à $41^{\circ}$ de latitude sud), les vignobles chiliens ont attiré en 2015 quelque 600000 visiteurs - chiliens (17\%) mais surtout étrangers (de diverses nationalités, dont $40 \%$ du Brésil) ; chiffre certes encore bas comparé à d'autres pays du nouveau monde viticole ${ }^{10}$, mais qui représente une augmentation de $10 \%$ par rapport à 2014 , après une croissance de $19 \%$ entre 2012 et 2015 (site Internet todovinos.cl, 2016), et qui a presque multiplié par trois le nombre de visiteurs de 2005 (237 225 visiteurs; voir Inalaf et al., 2012).

En $2013^{11}$, le pays possédait 78 caves ouvertes au tourisme, soit une augmentation progressive $\mathrm{e}^{12}$ et diversifiée depuis 2005 (+ M Consultores, 2014 ; site todovinos.cl, 2016). En effet, le produit œnotouristique actuel offert par ces vignobles est considérablement différent de celui des années 1990 (une visite de 30 minutes incluant un passage par les caves), les circuits ayant évolué dans leur durée et dans leur contenu (Zamora et Barril, 2007). La plupart des 78 caves et vignobles ouverts au tourisme (82\%) proposent toute une gamme de visites et de prestations, de la plus économique à la plus onéreuse. Parmi celles-ci, figurent en bonne place - depuis 2005 - l'achat de $\operatorname{vin}^{13}$, les dégustations, les visites en caves et chais, et des promenades à travers les vignes, réalisées via des touropérateurs dans $90 \%$ des cas (+M Consultores, 2014). Par ailleurs, 35 de ces 78 vignobles proposent également des services d'alimentation ( $44,9 \%)$ pour du tourisme gastronomique, 16 font aussi hébergement $(20,5 \%), 68$ possèdent une salle de dégustation (87,2\%), 66 une boutique (75,8\%), 57 un point d'exposition ( $73 \%$ ) et 44 ont installé des belvédères $(56,4 \% ;+M$ Consultores, 2014). L'œnotourisme se marie aussi bien avec des activités liées à la santé (vinothérapie), à la nature (parcours autour des vignes), à la culture (musée du vin), à l'écologie (architecture et production durables), et aux loisirs de tourisme (calèches et même téléphériques pour parcourir les vignobles). En 2016, le Chili compte quatre grandes routes du vin à forte activité entretenues par des ressources publiques et privées. Cette même année, le nombre de caves ouvertes au tourisme serait monté à $94^{14}$ (soit une augmentation de $19 \%$ par 
rapport à 2013), dont $65 \%$ de type familial et de petite taille, avec 20,6\% de visiteurs en plus entre 2014 et 2015 (Infyde, 2016).

Ces constats correspondent aux modèles dominants d'œnotourisme au niveau mondial, avec visites des caves et des domaines (Inalaf et al., 2012). Le Chili adopte ainsi la stratégie dite du nouveau monde viticole (Lignon-Darmaillac, 2014), qui se concentre sur d'autres éléments et expériences touristiques: la culture locale identitaire, une approche "high-tech» des domaines et des caves modernes qui embrassent les développements technologiques et architecturaux, les services de dégustation et de restauration. Cela par opposition à la stratégie européenne, celle du vieux monde viticole dont la «culture du vin » est enracinée dans une longue histoire culturelle (Zamora et Barril, 2007 ; Inalaf et al., 2012 ; Lignon-Darmaillac, 2014 ; Infyde, 2016); pourtant, au Chili, certaines caves traditionnelles, très anciennes, entrent aussi dans le jeu et peuvent se targuer de posséder des pieds de vignes centenaires (Inalaf et al., 2012). Ainsi, l'œnotourisme est devenu un outil de marketing (national et international) reconnu et une source de revenus supplémentaires pour les vignobles ${ }^{15}$, tout en diversifiant l'offre touristique du pays, portant ainsi des enjeux économiques de plus en plus significatifs $(0,6 \%$ du tourisme du pays et $0,013 \%$ de son PIB en 2013 ; Infyde, 2016).

\section{Le processus d'émergence du paysage culturel viticole chilien - entre valorisation patrimoniale et commerciale/œnotouristique}

21 L'exemple des paysages viticoles chiliens est emblématique du passage progressif d'une mise en relation à une mise en valeur d'une production agricole avec son paysage, et donc de l'émergence - voire de la patrimonialisation - d'un paysage culturel dans le pays. Cela suit la logique des processus d'inscription au patrimoine engagés par des pays européens comme la France, où la notion du patrimoine rural, associée au bâti agricole et apparue dans les années 1980, a évolué pour accompagner la labellisation de certains produits, s'étendant au paysage agricole dans sa totalité dans les années 1990 (Chevallier et al., 2000 ; Dérioz, 2013). Des initiatives de mise au patrimoine viticole existent également en France depuis la première moitié $\mathrm{du} \mathrm{xx}^{\mathrm{e}}$ siècle (LignonDarmaillac, 2014).

Cependant, dans le cas chilien nous constatons que la figure de paysage culturel ici exposée émerge: (a) sans cadre normatif, et (b) dans une démarche particulière d'appropriation et de valorisation des paysages du vignoble, associée à la mise en valeur de son patrimoine comme stratégie de promotion viticole et œnotouristique. Ainsi, est-il intéressant d'analyser la façon dont le paysage est pris en compte par les trois groupes d'acteurs - le secteur privé, le secteur public et le secteur universitaire qui, en résonance, ont élaboré l'idée de paysage viticole patrimonial en la positionnant comme une question cruciale au Chili.

Le prélude et le tournant de ce processus est une initiative lancée dès $2011^{16}$ par divers organismes publics emmenés par la Direction des bibliothèques archives et musées du pays (Dibam) du ministère de l'Éducation avec l'appui du ministère de l'Agriculture, le CMN et soutenue par le secteur universitaire et des associations de producteurs, afin de mettre en valeur le patrimoine vitivinicole du pays. Cette coopération a abouti à la 
création de la Commission du patrimoine vitivinicole chilien en 2012 ainsi qu'à l'initiative conduite par la Bibliothèque nationale du Chili de présenter un dossier visant à faire inscrire le paysage viticole chilien sur la liste du patrimoine de l'Unesco, suivant l'exemple des paysages culturels viticoles déjà classés (six vignobles historiques d'Europe déclarés entre 1997 et 2007), ainsi que celui de la candidature au paysage culturel du vin de La Rioja et Rioja Alavesa en Espagne (encore non classé). Cette initiative, soutenue par des experts internationaux ${ }^{17}$, cherche à faire reconnaitre, d'une part, la diversité unique au monde des paysages viticoles émaillant les vallées du nord au sud du Chili, comme autant de "cartes postales » caractéristiques du pays; et, d'autre part, l'ancienneté et l'importance de cette culture dans l'histoire du pays et ses caractéristiques particulières ${ }^{18}$ (Fundación de Comunicación, Formación y Cultura del Agro [FUCOA], 2012). La protection du paysage culturel à vocation patrimoniale est enfin une question posée sur la scène nationale.

Par la suite et de façon concomitante, différentes stratégies de positionnement et de promotion de la viticulture et de l'œnotourisme chiliens, via la coopération d'organismes publics et privés, en lien avec des études universitaires sur le sujet, cherchent à mettre en valeur les caractéristiques des vignobles du Chili. Parmi celles-ci, sont considérés comme des atouts: une diversité et une spécificité géographiques uniques au monde (extension latitudinale et altitudinale) ; une synthèse représentative du nouveau et de l'ancien monde (superposition de l'héritage hispanique traditionnel et de l'archétype du vignoble français, entre autres; Rojas, 2015 ; Tironi et Aravena, 2015); une longue histoire viticole au sein du nouveau monde avec des vignobles centenaires (Muñoz, 2015); certaines pratiques œnologiques traditionnelles (en particulier dans les vallées méridionales du pays; Ledesma, 2015); une importante viticulture biologique ${ }^{19}$; et l'une des meilleures productions viticoles au monde. Or, à tous ces atouts favorables au développement de la viticulture et du produit œnotouristique chilien s'ajoute depuis peu la prise en considération du paysage culturel viticole du pays.

Ainsi, la littérature récente sur la viticulture chilienne indique que celle-ci n'aurait pas seulement été le principal élément configurateur du paysage culturel de la zone centrale à travers les siècles - la beauté du vignoble donnant "une impression d'austère élégance au paysage chilien » (Rojas, 2015) -, mais qu'elle ferait même partie du développement historique de son patrimoine matériel et immatériel, tout comme de la construction de l'identité nationale (Lacoste, 2005 ; Rojas, 2014 ; Rojas, 2015). Dans Patrimonio vitivinícola, ouvrage collectif ${ }^{20}$ portant sur le patrimoine vitivinicole du pays, il est dit que les paysages font partie de la culture du vin, au même titre que les pratiques traditionnelles et la diversité viticole (Tironi et Aravena, 2015). On y trouve également des images légendées qui présentent des "paysages » de vallées, ainsi que des allusions à l'impact positif sur les paysages de certaines variétés en automne (Solminihac, 2015; Pszczólkowski, 2015 voir figure 1b), aux divers paysages vitivinicoles sur toute la longueur du territoire chilien et à leur beauté naturelle (Solminihac, 2015). Toutefois, dans cet ouvrage, le paysage reste anecdotique parmi les éléments mentionnés, matériels et immatériels, du patrimoine viticole, le focus étant mis sur les aspects historiques de la viticulture, des techniques agronomiques et de certaines variétés: éléments qui, certes, font partie des paysages culturels, mais auxquels ces derniers ne se réduisent pas. En ce sens, cette prise en compte du paysage n'approfondit pas davantage celle mise en avant par certains auteurs il y a quelques 
décennies, qui évoquait alors des vues panoramiques pour accompagner les photographies (cf. Alvarado Moore, 1985 ; figure 1).

Figure 1. Images des paysages viticoles dans deux ouvrages chiliens dédiés à la viticulture, à 30 ans d'écart

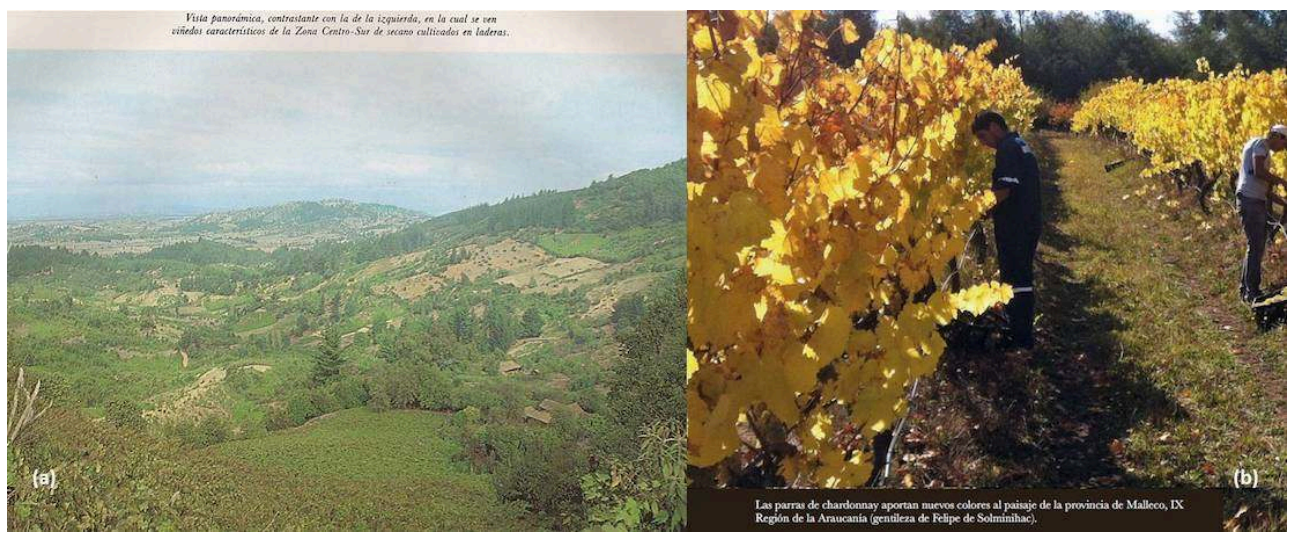

Sources images : (a) Alvarado Moore dans son ouvrage Chile, Tierra de Vino de 1985 ; (b) Solminihac dans l'ouvrge Patrimonio vitivinícola de 2015.

D'autre part, les paysages viticoles du pays sont mentionnés dans les rapports les plus récents concernant le potentiel mais aussi les défis que rencontre l'activité œnotouristique dans le pays. Ainsi, dans le rapport « Feuille de route et diagnostic du programme "Enotourisme durable de la zone centrale" " (Infyde, 2016) - commandé par le programme public de promotion Enoturismo Chile et Transforma Alimentos ${ }^{21}-$, le paysage culturel du vin est cité comme un atout pour l'œnotourisme du pays, notamment en ces termes: « Paysage culturel viticole d'importance, où convergent des facteurs naturels, culturels et humains autour de la viticulture ». De même, dans des études sorties du secteur universitaire sur le développement de l'œnotourisme au Chili (Godoy et Meza, 2014 ; Inalaf et al., 2012), les paysages viticoles sont nommés parmi les facteurs d'intérêt des différentes vallées du pays ("paysage unique", "paysage attractif ", "paysage en harmonie »), mais aussi en tant qu'élément à prendre en compte dans ce type d'activité touristique selon les références internationales en la matière (notamment françaises).

S'ajoute à ce processus la reconnaissance à l'international de certaines initiatives œnotouristiques chiliennes où le paysage viticole se dessine également, bien encadré par les montagnes. On peut citer la vallée de Colchagua ${ }^{22}$, laquelle est apparue en 2014 parmi les dix premières destinations de lune de miel dans l'édition de Lonely Planet's Best honeymoon destinations for 2014 («incroyablement magnifique, avec les sommets enneigés des Andes s'élevant au-dessus des collines tapissées de vignes »; site Internet lonelyplanet.com, 2014); et ses pistes cyclables au milieu des vignobles sont parmi les dix plus agréables au monde en 2015, selon The Guardian, traversant «un paysage dramatique surplombé par les hautes montagnes de la cordillère des Andes, où des cow-boys chiliens [...] parcourent des collines vert émeraude [...]» (site Internet theguardian.com, 2015). Ailleurs, la vallée d'Elqui figure en 2015 sur la liste des « douze paysages viticoles les plus impressionnants au monde » (à la $10^{\mathrm{e}}$ place) de la revue espagnole Vinopack, qui la décrit comme «l'un des plus beaux paysages du pays » (site Internet Vinopack.es, 2015) (voir figure 2). 
Figure 2. Le paysage viticole de la vallée d'Elqui souligné dans le site Internet de la revue espagnole Vinopack

10. Valle del Elqui - Chile

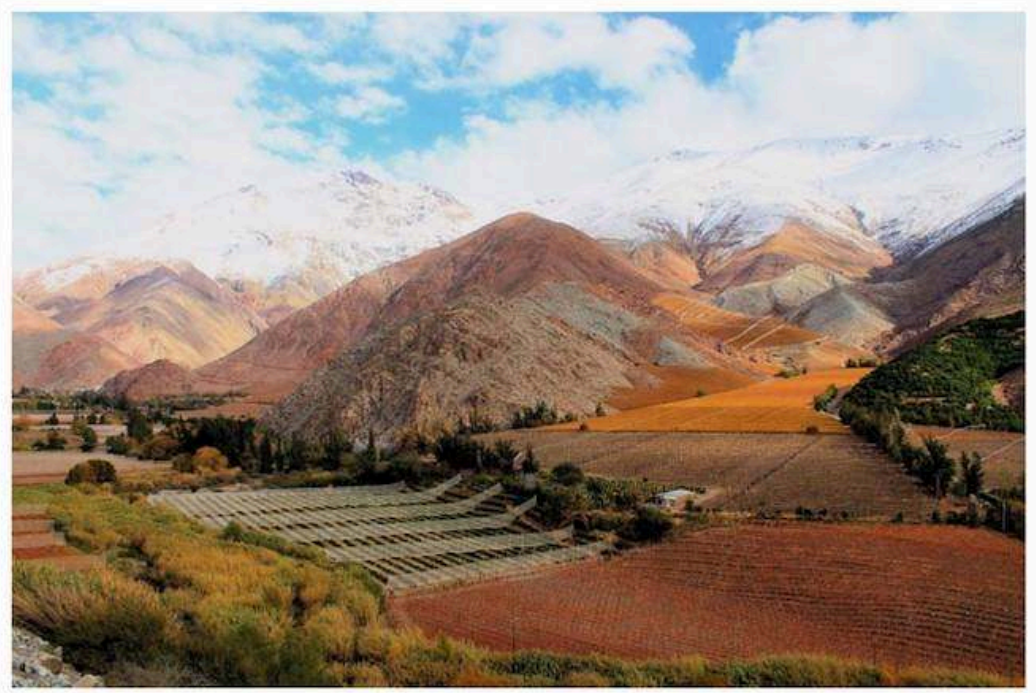

Foto: Aguslepe

El valle pertenece a la Región de Coquimbo y es uno de los paisajes más bellos del país. Se encuentra a 500 kilómetros de Santiago, conformando la frontera norte de las regiones vinícolas de Chile.

Source image : Aguslepe vue sur le site InternetVinopack.es 2017 (http://www.vinopack.es/los-12paisajes-de-vinedos-mas-impresionantes).

Dès lors, les stratégies actuelles de promotion publiques et privées semblent partie prenante de cette prise de conscience paysagère en parallèle à l'essor de l'œnotourisme. La place du paysage comme élément de marketing utilisé en 2017 par les sites Internet des vignobles - leur principal moyen de promotion (68-71 vignobles en possèdent en 2013 ; + M Consultores, 2014) - en est une première piste ${ }^{23}$. On prendra pour exemples les deux sites officiels de promotion des services publics du tourisme ${ }^{24}$, ainsi que ceux des 26 producteurs (caves ${ }^{25}$ ) possédant des sites Internet et installés dans la vallée la plus fréquentée du pays : la vallée du fleuve $\mathrm{Maipo}^{26}$ dans la région viticole Valle Central ${ }^{27}$ (figure 3). 
Figure 3. Localisation de la vallée du Maipo dans la zone centrale du Chili

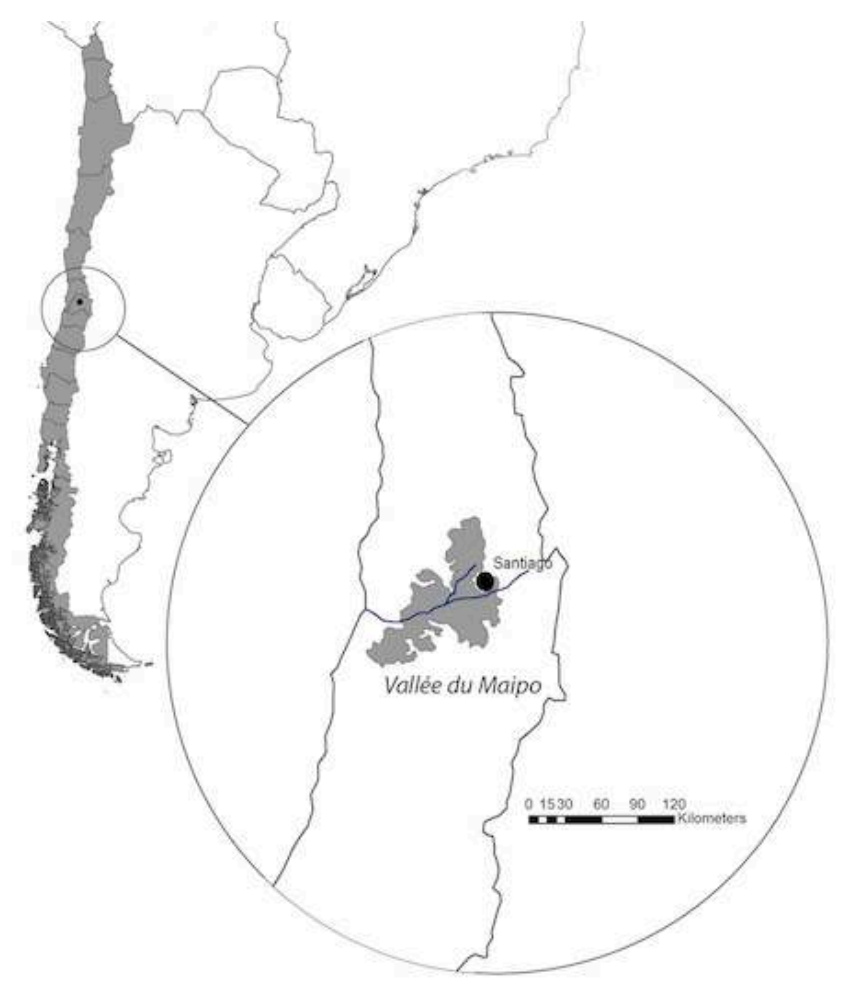

À l'exception de l'un des sites de caves, tous les sites Internet analysés présentent des photographies de paysages (voir figure 4), dans certains cas classées dans des galeries avec de nombreuses images. Pour ces 25 sites de caves, les photographies de paysages viticoles sont montrées souvent dans les rubriques "vignobles", "terroir» ou « vallées », que possèdent 21 des 25 sites ( $84 \%$ ) et sur la page d'accueil pour 15 sites (60\%). Sur ce total ( 25 sites de caves +2 sites officiels de tourisme), 19 sites $(70,4 \%)$ montrent uniquement des images de paysages viticoles; 7 (25,9\%) des paysages de vignes et aussi naturels (nature parfois très éloignée de la région viticole concernée, plus proche d'une image écotouristique, comme le haut plateau au nord des Andes montré dans la figure $4 \mathrm{~b})$; et un seul $(3,7 \%)$ montre une unique image de paysage naturel (de cordillère). Dans la plupart de ces images, sont mis en avant les aspects agronomiques (de vieux pieds, leurs feuilles, leur alignement, voir figures $4 \mathrm{l}$ et $4 \mathrm{e}$ ) et esthétiques des vignobles (des vignes en automne, voir figures $4 \mathrm{c}$ et $4 \mathrm{i}$ ); ces derniers sont le plus souvent encadrés par les montagnes ou encaissés dans la vallée (voir figures $4 \mathrm{a}, \mathrm{c}, \mathrm{f}, \mathrm{g}, \mathrm{h}, \mathrm{i}, \mathrm{j}, \mathrm{k}, \mathrm{m}$ et $\mathrm{n}$ ), avec souvent des arbres parmi les parcelles, ou bien bordés par ou avec des « îlots » de végétation naturelle (figure $4 \mathrm{e}, \mathrm{f}, \mathrm{g}, \mathrm{h}, \mathrm{m}$ et $\mathrm{n}$ ).

Parmi les différents éléments de marketing mis en avant (« discours publicitaires ») par les caves étudiées, mais aussi sur les sites Internet officiels du tourisme, dominent principalement les allusions à la situation géographique des vallées, leur qualité idéale pour des cépages déterminés et les atouts naturels des terroirs (climat, microclimat, le fleuve et son lit, proximité de la mer, diversité des sols, altitude, force des vents) - avec un élément qui ressort invariablement : la cordillère des Andes, référence en images et en discours, comme décor pour les vignobles ${ }^{28}$. Prépondérantes également, les allusions à la production durable de la culture, aux vignes biologiques ${ }^{29}$, ainsi qu'à la relation agriculture-nature, et pas seulement pour les domaines labélisés. Tout comme sur les 
images, cette nature qui entoure les vignobles est assez présente ( lieu unique, protégé par la nature ", vignobles "cernés par les chaînes de montagnes et la rivière Maipo ", «l'environnement naturel qui les entoure »), parfois sublimée (« entre les montagnes provocatrices et une mer sans fin»), avec des allusions à ses qualités protectrices, de pureté garante de biodiversité. Dans cette nature perçue «en dehors » des vignobles, semble réapparaître la division conceptuelle et législative entre paysage naturel et paysage culturel que nous avons évoquée dans la première partie, bien que la synergie établie ici entre les deux domaines les rapproche de l'idée de paysage culturel.

Le mot « paysage » est utilisé explicitement par un seul des sites de tourisme officiels et par 11 des sites Internet de caves de toutes tailles $(42,3 \%)$, avec des stratégies marketing différentes. Sur les sites où le terme lui-même est absent, le paysage peut apparaître de façon implicite lorsqu'il est fait référence aux belvédères qui jalonnent les circuits de 7 caves ( « une vue panoramique sur le vignoble »). Le terme paysage n'est pas développé en profondeur, étant utilisé surtout dans son acception esthétique, voire contemplative ("profitez du magnifique paysage qui entoure notre cave»), en lien étroit avec les circuits œnotouristiques et donc dans la rubrique dédiée au tourisme (plusieurs domaines proposent des randonnées dans les vignes et des parcours dans les sites naturels environnants). Seuls quatre sites mentionnent le terme en lien avec des éléments du terroir ("des vignobles ancestraux dominent encore le paysage »). En outre, ce paysage mentionné peut être de type naturel avec ses espèces autour des vignobles («profitez du paysage unique de Colchagua, en observant la diversité de la flore et de la faune autochtone ", "un paysage où règnent les cactus »). Dans une moindre mesure, il est nommé et reconnu comme un paysage (culturel) du vignoble ( " profitez d'un beau paysage campagnard », « profitez du paysage viticole », « admirez depuis votre terrasse les magnifiques paysages des vignes alentour »), dont le caractère alliant nature et viticulture peut être aussi souligné (« voyez comme la forêt se marie avec le paysage. Forêt et vignes se confondent »). La catégorie du paysage culturel commence ainsi à trouver sa place dans ces terroirs. 
Figure 4. Quelques photographies de paysages viticoles extraites des sites Internet chiliens de promotion viticole et œnotouristique analysés

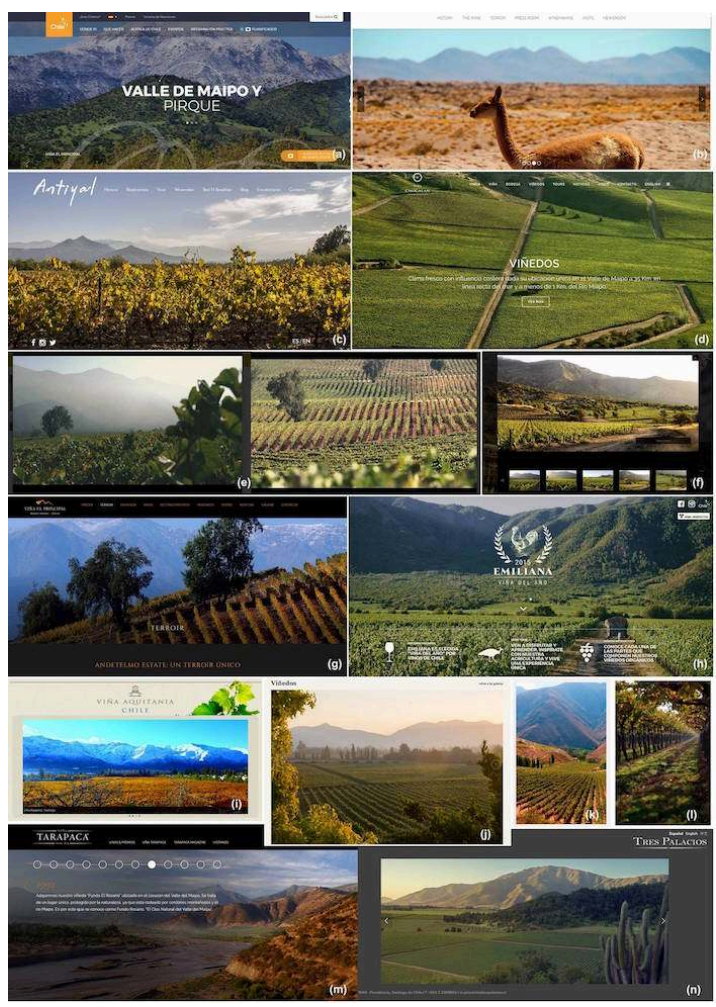

Sources images = (a) : http://chile.travel/intereses-destacados/rutas-del-vino/valle-de-maipo-y-pirque (๔ Chile Travel 2017) ; (b) : http://www.almavivawinery.com/en/terroir (@ Viña Almaviva 2017) ; (c) : http://antiyal.com/es/welcome (@ Viña Antiyal 2017) ; (d) : https://chocalanwines.com/web/vinedos (@ Viña Chocalán 2017) ; (e) : http://www.conosur.com/vineyards-winery/our-valleys [capture vidéo] (๔) Viña Cono Sur 2017) ; (f) : http://www.santarita.com/chile/vinedos-bodegas/vinedos/maipo (C Viña Santa Rita 2017) ; (g) : https://www.elprincipal.cl/terroir.html (@ Viña El Principal 2017) ; (h) http://www.emiliana.cl/\#Emiliana (@ Viña Emiliana 2017) ; (i) : http://aquitania.cl/en (@ Viña Aquitania 2017) ; (j) : http://odfjellvineyards.cl/es/galeria-de-imagenes/vinedos (@ Viña Odjfjell 2017) ; (k) : http:// www.demartino.cl/terrunos.php (@ Viña De Martino 2017) ; (I) : http://www.montgras.cl/en/vineyards/ intriga-vineyard (@ Viña MontGras 2017) ; (m) : http://www.tarapaca.cl/historia (@ Viña Tarapacá 2017) ; (n) : http://www.vinatrespalacios.cl/vinedo/(CViña tres palacios 2017)

De cette manière, et d'après cet échantillon, une certaine image du paysage viticole distinct du paysage naturel environnant ou en synergie avec lui - semble devenir un élément de plus en plus essentiel de l'image promue et projetée dans les outils de marketing des sites Internet de promotion viticole publics comme privés. L'exploitation publicitaire des paysages viticoles est en effet l'une des tendances internationales dans l'évolution du produit œnotouristique (Infyde, 2016), mais ici le paysage reste peu défini et donc peu exploité. Certes, le contexte est différent (électronique) et le medium présente certaines particularités par rapport aux enquêtes de terrain - notamment le locuteur est inconnu. Pour autant la démarche repose sur un même ensemble de principes (Pastinelli, 2011), bien que nous ne soyons pas en mesure d'indiquer l'origine des contenus iconographiques et des lexiques analysés, ni de confirmer qu'ils expriment effectivement la vision des agriculteurs ou des entreprises agricoles. Nous ne pouvons pas dire non plus s'ils reproduisent ou s'inspirent ici d'un autre modèle Internet national ou international. Cependant, il s'agit là de l'image que l'on cherche à donner des domaines viticoles pour promouvoir la production viticole ainsi que le tourisme associé ; et celle-ci montre une tendance à déplacer l'attention du vin (l'image 
d'accueil de certains sites) vers le terroir, le paysage - même s'il peut s'agir du paysage de la vigne lui-même ou alors du paysage cadrant les vignes.

\section{Conclusion}

33 Les paysages culturels ont longtemps été absents du cadre législatif et discursif de la protection des paysages au Chili, lequel est dominé depuis presque un siècle par une attention aux systèmes naturels. Dans la mesure où l'idée de patrimonialisation des paysages façonnés par l'homme est reconnue sur la scène internationale, des modèles de protection ont été proposés ces dernières décennies sans pour autant arriver encore à leur établissement normatif dans le pays.

L'importance économique de la viticulture chilienne, et la reconnaissance qu'elle a acquise comme produit-image du pays à l'étranger, s'est traduite ces dernières années par l'adoption d'une perspective différente sur ces territoires, focalisée dans leur promotion commerciale et touristique via des efforts publics et privés. Au cours de cette évolution, et pendant le double processus de recherche des éléments propres de différenciation et de références mondiales du produit viticole et œnotouristique, de nouveaux éléments du patrimoine viticole chilien ont émergé, notamment les paysages culturels du vignoble. En effet, il a été déjà indiqué qu'à travers son offre touristique culturelle, l'œnotourisme peut non seulement contribuer à la patrimonialisation des traditions vigneronnes, mais aussi faire naitre de nouvelles formes de patrimoines (Lignon-Darmaillac, 2014), comme les paysages. Les vignobles sont ainsi un exemple emblématique de patrimonialisation paysagère de l'agriculture au Chili.

Nous l'avons vu, l'émergence de cette catégorie " paysage culturel viticole " au Chili se produit en l'absence d'un cadre national normatif de protection, et donc elle semble davantage venue de l'extérieur, en comparaison avec d'autres pays viticoles du monde, notamment avec leurs stratégies commerciales (œnotouristiques) ainsi que leurs processus de mise au patrimoine (comme les paysages culturels viticoles classés au titre du patrimoine mondial). Cela expliquerait que cette catégorie reste encore floue et vide de contenu, ce que l'on constate :

- dans l'absence de description des paysages viticoles chiliens en tant que tels et le peu d'éléments donnés pour détailler en termes académiques mais aussi commerciaux leurs spécificités paysagères - outre leur présence, leur diversité biogéographique et leur importance - car comme l'indique Yves Luginbühl (2005) « il n'y a pas un paysage viticole, mais une immense variété de paysages de vignes »;

- dans l'emphase portée sur le paysage naturel des terroirs, associé à un territoire géographique, avec des particularités environnementales, telles que l'indiquent nos résultats également signalés par d'autres auteurs (Rojas, 2015). Ces paysages naturels sont l'arrière-plan, les décors des vignobles, auxquels ils apportent qualité et durabilité, constituant une ressource présente et mobilisée dans les discours de valorisation et de marketing ainsi que dans les activités œnotouristiques des vignobles chiliens.

Les paysages naturels environnant les vignobles sont positivement valorisés par les visiteurs (Johnson, 1998 et Getz, 2000 cités par Godoy et Meza, 2014). Dans le cas chilien, outre leur protection législative retracée dans cet article, il faut considérer l'importance des beautés naturelles dans la construction des symboles touristiques nationaux (Booth, 2008), également reconnus au niveau international (la Patagonie, le désert d'Atacama). C'est peut-être davantage cette nature que l'on cherche à mettre en 
avant sur les terres de vignobles: la Cordillère des Andes, la flore et la faune autochtones.

Ainsi, nous confirmons ce que Faliet avait indiqué il y a dix ans concernant le paysage viticole chilien : il s'agit d'un paysage à complexité culturelle et culturale, relativement récent, en quête d'identité (Faliet, 2005).

L'évolution de la prise en compte de ce type de paysages, en phase avec le processus de mise en protection d'autres pays, pourrait initier une discussion sur les paysages culturels au Chili, avec une attention de plus en plus forte portée sur les paysages produits par la vigne et leurs caractéristiques paysagères propres (architecture, hauteur et densité de rangs de la vigne, présence de réseaux verts, d'arbres isolés ou de bosquets, types de divisions parcellaires, forme et taille des parcelles, types d'aménagement du sol pour en citer quelques-unes ${ }^{30}$ ). Ces éléments peuvent en premier lieu fournir des outils pour appréhender l'originalité de ces paysages (Joliet, 2005), ainsi que nous donner des pistes concernant l'intensivité de la culture ou, au contraire, quelques atouts concernant sa durabilité (les clichés montrant des arbres entre les vignobles sur les sites Internet nationaux ne sont pas anodins).

En effet, en exposant le territoire comme un tout (culture, paysage et services ; Zamora et Barril, 2007), l'œnotourisme et les routes du vin pourraient, par exemple, avoir des répercussions positives sur la planification et le développement durable du territoire (voir le vade-mecum de l'œnotourisme européen, Assemblée des régions européennes viticoles [AREV], 2005). Ce dernier aspect acquiert une importance essentielle si nous considérons une planification intégrale du territoire qui prenne en compte les enjeux paysagers des évolutions contemporaines de la culture dans le pays (expansion, augmentation de l'irrigation): des paysages viticoles intensifs ou bien extensifs, notamment, sont-ils perçus de façon équivalente sur le plan esthétique? En ce sens, des choix sont à effectuer entre la patrimonialisation et la mise en protection des attributs valorisés des paysages viticoles, en vue de leur appréciation touristique, et les formes de gestion destinées uniquement à une augmentation de la production (principalement pour l'exportation). C'est grâce à ce type de débat que peuvent se mettre en place les fondations d'une politique de protection du paysage culturel au Chili.

\section{BIBLIOGRAPHIE}

+ M Consultores, « Catastro del Enoturismo 2013 », Vinos de Chile, Santiago, Subsecretaría de Turismo, Sernatur et CORFO, 2014.

Alvarado Moore, R., Chile tierra del vino - Chile land of wine, Santiago, Editorial Lord Cochrane, 1985, $88 \mathrm{p}$.

Ambroise, R., « Les pressions et les enjeux paysagers concernant les sites viticoles ", dans Conseil international des monuments et des sites (Icomos), Étude thématique : les paysages culturels viticoles dans le cadre de la Convention du patrimoine mondial de l'Unesco, Paris, Éditions Icomos, 2005, p. 51-55. 
Aravena, R. (ed.), Patrimonio vitivinícola. Aproximaciones a la cultura del vino en Chile, Santiago, Ediciones Biblioteca Nacional, 2015, 297 p.

Assemblée des régions européennes viticoles (Arev), « Vademecum de l'œEnotourisme Européen », projet Vintur, 2005.

Booth, R., « Turismo y representación del paisaje. La invención del sur de Chile en la mirada de la Guía del Veraneante (1932-1962) », Nuevo Mundo Mundos Nuevos, « Débats », février 2008, URL :http://nuevomundo.revues.org/25052.

Carbonneau, A., « Architecture de la vigne et paysage », dans Conseil international des monuments et des sites (Icomos), Étude thématique : les paysages culturels viticoles dans le cadre de la Convention du patrimoine mondial de l'Unesco, Paris, Éditions Icomos, 2005, p. 31-40.

Chevalier, D., Chiva, I. et Dubost, F., « L'invention du patrimoine rural », dans Chevalier, D. (dir.), Vives Campagnes. Le patrimoine rural, projet de société, Paris, Éditions Autrement, coll. « Mutations », no 194, 2000, p. 11- 55.

Conseil international des monuments et des sites (Icomos), Étude thématique : les paysages culturels viticoles dans le cadre de la Convention du patrimoine mondial de l'Unesco, Paris, Éditions Icomos, 2005, $175 \mathrm{p}$.

Consejo de Monumentos Nacionales (CMN), « Normas sobre Zonas Típicas o Pintorescas », "Cuadernos del Consejo de Monumentos Nacionales», Segunda Serie, n 37, Santiago, Marzo 2001, URL : http://www.unesco.org/culture/natlaws/media/pdf/chile/

chil_normas_zonas_pitorescas_spaorof.pdf.

Corporación Nacional Forestal (CONAF), « Plan de Manejo Parque Nacional Vicente Pérez Rosalez », Puerto Montt, Departamento Áreas Protegidas, Región de Los Lagos. Surambiente Limitada, 2015, 299 p.

Del Pozo, J., Historia del vino chileno : desde 1850 hasta hoy, Santiago, Editorial Universitaria, 1999, $321 \mathrm{p}$.

Dérioz, P., « La place du paysage dans l'action des parcs naturels régionaux depuis la loi Paysage (1993) : enjeu central mais mission impossible? », Projets de Paysage, $n^{\circ} 9$, décembre 2013, URL : https://www.projetsdepaysage.fr/fr/

la_place_du_paysage_dans_l_action_des_parcs_naturels_regionaux_depuis_la_loi_paysage_1993_

Faliet, C., « Le paysage viticole chilien », dans Conseil international des monuments et des sites (Icomos), Étude thématique : les paysages culturels viticoles dans le cadre de la Convention du patrimoine mondial de l'Unesco, Paris, Éditions Icomos, 2005, p. 79-80.

Fundación de Comunicación, Formación y Cultura del Agro (FUCOA), «El paisaje vitivinícola chileno como Patrimonio de la Humanidad », Santiago, Sección Nuestra Tierra, 7 noviembre de 2012, URL : http://www.fucoa.cl/noticias-nuestra-tierra/el-paisaje-vitivinicola-chileno-comopatrimonio-de-la-humanidad/

Godoy, S. et Meza, C., « Bases para el desarrollo del negocio enoturístico en el Valle de Almahue », Santiago, Seminario para optar al Título de Ingeniero Comercial, Facultad de Economía y Negocios, Universidad de Chile, 2014, 126 p.

Inalaf, M., Ogalde, O., et Verdugo, M., « Enoturismo en Chile : una oportunidad de desarrollo y crecimiento para las viñas », Santiago, Seminario para optar al Título de Ingeniero Comercial, Facultad de Economía y Negocios, Universidad de Chile, 2012, 104 p. 
Infyde (Información y Desarrollo S.L.), « Servicio de Consultoría Experta para el Diseño de la Hoja de Ruta de Enoturismo Chile », Santiago, Programa Estratégico Meso Regional de Especialización Inteligente « Enoturismo Sustentable de la Zona Central », Enoturismo Chile, 2016, 196 p.

Instituto de la Patagonia, Icomos et Universidad de Magallanes, «Presentación Encuentro Paisajes Culturales en Patagonia », Punta Arenas, 12 al 14 de septiembre 2013, URL : http:// www.equiponaya.com.ar/eventos/jornadas\%20ICOMOS\%202013.htm

Joliet, F., «Typologie plastique des paysages de vigne », dans Conseil international des monuments et des sites (Icomos), Étude thématique : les paysages culturels viticoles dans le cadre de la Convention du patrimoine mondial de l'Unesco, Paris, Éditions Icomos, 2005, p. 23-29.

Lacoste, P., « El vino y la nueva identidad de Chile », Revista Universum, vol. 2, n² 20, 2005, p. 24-33.

Ledesma, J.-J., « Itata y Biobío, el tiempo detenido », dans Aravena, R. (ed.), Patrimonio vitivinícola. Aproximaciones a la cultura del vino en Chile, Santiago, Ediciones Biblioteca Nacional, 2015, p. 106-113.

Lignon-Darmaillac, S., « L'œnotourisme, redécouverte des valeurs patrimoniales des vignobles historiques, développement des vignobles du nouveau monde ", Culture (Revista de Cultura e Turismo), édition Spéciale « Vin, Patrimoine, Tourisme et développement : convergence pour le débat et le développement des vignobles du monde ", année 8, n³ 3, 2014, p. 30-46.

Luginbühl, Y., " Paysages viticoles », dans Conseil international des monuments et des sites (Icomos), Étude thématique : les paysages culturels viticoles dans le cadre de la Convention du patrimoine mondial de l'Unesco, Paris, Éditions Icomos, 2005, p. 15-18.

Mata Olmo, R., « Agricultura, paisaje y gestión del territorio », Polígonos, Revista de Geografía, n 14, 2004, p. 97-137.

Ministerio de Agricultura, « Política Nacional de Desarrollo Rural 2014-2024 », Santiago, Comité Técnico Interministerial, 2014, 32 p.

Miranda, M., Flores, L., Reyes, S., Mashini, D., Misleh, D., Bettancourt, P., « Valorización de los vínculos urbanos, rurales y silvestres en la generación de instrumentos de planificación territorial integrada », dans Irarrázaval, I., Pozo, C. et Letelier, M. (ed.), Propuestas para Chile, Concurso Políticas Públicas 2015, Santiago, Ediciones Centro de Políticas Públicas Pontificia Universidad Católica de Chile, 2015, p. 171-198.

Muñoz, J.-G., « Viñas actuales en los mismos terrenos que en los siglos XVI y XVII », dans Aravena, R. (ed.), Patrimonio vitivinícola. Aproximaciones a la cultura del vino en Chile, Santiago, Ediciones Biblioteca Nacional, 2015, p. 34-47.

Nahrwold, H., Vinos de Chile, Santiago, Editorial Contrapunto, 2014, 284 p.

Oficina de Estudios y Políticas Agrarias (ODEPA), « Expansión Urbana y suelo Agrícola : Révision de la Situation en la Région Metropolitana », Santiago, Ministerio de Agricultura, Gobierno de Chile, 2013, URL : http://www.odepa.cl/wp-content/files_mf/1387811651expansionUrbana.pdf

Oficina de Estudios y Políticas Agrarias (ODEPA), « Estadísticas productivas : estadísticas de vides, Santiago, Ministerio de Agricultura », Gobierno de Chile, 2017a, URL : http://www.odepa.gob.cl/ estadisticas/productivas/

Oficina de Estudios y Políticas Agrarias (ODEPA), « Catastro vitícola nacional, Santiago, Ministerio de Agricultura, Gobierno de Chile », 2017b, URL : http://www.odepa.gob.cl/

documentos_informes/catastro-viticola-nacional/ 
Pastinelli, M., « Pour en finir avec l'ethnographie du virtuel ! : Des enjeux méthodologiques de l'enquête de terrain en ligne », Anthropologie et Sociétés, vol. 35, nºs 1-2, 2011, p. 35-52.

Pszczólkowski, P., « Carmenère, mayoría de edad (1994-2014) : la recuperación de una variedad única ", dans Aravena, R. (ed.), Patrimonio vitivinícola. Aproximaciones a la cultura del vino en Chile, Santiago, Ediciones Biblioteca Nacional, 2015, p. 50-55.

Rojas, G., « Patrimonio e identidad Vitivinícola. Reflexiones sobre la evolución de los significados culturales del vino en Chile », Rivar, vol. 2, n 4, IDEA-USACH, 2015, p. 88-105.

Rojas, G., « Identidad y Patrimonio Vitivinícola : Bases para el desarrollo del turismo cultural del vino en Chile », Santiago, Documento de trabajo Facultad de Economía y Negocios, Universidad de Chile, 2014, URL : https://www.academia.edu/12883287/

Identidad_y_Patrimonio_Vitivin \%C3 \%ADcola_Bases_para_el_desarrollo_del_turismo_cultural_del_vino_en_Chile Servicio Nacional de Turismo (Sernatur), « Cuadros estadísticos : Llegada de turistas extranjeros Marzo 2017 », Santiago, marzo 2017, URL : https://www.sernatur.cl/estadisticas/.

Solminihac, F. de, « Dinamismo de la vitivinicultura chilena : el valle de Malleco », dans Aravena, R. (ed.), Patrimonio vitivinícola. Aproximaciones a la cultura del vino en Chile, Santiago, Ediciones Biblioteca Nacional, 2015, p. 58-64.

Tironi, A. et Aravena, R., « Presentación », dans Aravena, R. (ed.), Patrimonio vitivinícola. Aproximaciones a la cultura del vino en Chile, Santiago, Ediciones Biblioteca Nacional, 2015, p. 11-13. Unesco, « Report of the Expert Group on Cultural Landscapes », La Petite Pierre (France), 16th Session World Heritage Committee, 24-26 October 1992.

Zamora, J. et Barril, M. E., « Turismo y vino : un estudio formativo sobe la evolución de las rutas del vino en Chile », Estudios y Perspectivas en Turismo, n 16, 2007, p. 173-194.

\section{Sites Internet}

Site lonelyplanet.com, « Lonely Planet's Best honeymoon destinations for 2014 », Destinations, 2014, URL : https://www.lonelyplanet.com/travel-tips-and-articles/best-honeymoondestinations-for-2014/40625c8c-8a11-5710-a052-1479d277aefc

Site Theguardian.com, « Top 10 bike-friendly wine routes », Wine Holydays, Sunday 26 July 2015, URL : https://www.theguardian.com/travel/2015/jul/26/top-10-bike-friendly-wine-routes

Site Todovinos.cl, «Crece $10 \%$ el enoturismo en Chile, 600 mil visitas en 2015 », Sitio de Noticias e informaciones del mundo del vino, Sección Noticias, 1 de febrero 2016, URL : http:// www.todovinos.cl/wp2/2016/02/01/crece-10-el-enoturismo-en-chile-600-mil-visitas-en-2015/ Site Vinopack.es, «Los 12 paisajes de viñedos más impresionantes del mundo », Sección Lugares, 5 de enero 2015, URL : http://www.vinopack.es/los-12-paisajes-de-vinedos-mas-impresionantes

\section{ANNEXES}

Les 26 vignobles de la vallée du Maipo dont les sites Internet ont été analysés

\begin{tabular}{|l|l|l|l|l|l|l|}
\hline $\mathrm{N}^{\circ}$ & $\begin{array}{l}\text { Nom } \\
\text { Vignoble }\end{array}$ & $\begin{array}{l}\text { Année } \\
\text { fondation }\end{array}$ & $\begin{array}{l}\text { Vallées } \\
\text { viticoles }^{1}\end{array}$ & Site Internet & $\begin{array}{l}\text { Surface } \\
(\mathrm{Ha})\end{array}$ & Enotourisme $^{2}$ \\
\hline
\end{tabular}




\begin{tabular}{|c|c|c|c|c|c|c|}
\hline 1 & $\begin{array}{l}\text { Viña Concha } \\
\text { y Toro* }\end{array}$ & 1883 & $\begin{array}{l}\mathrm{Li}, \mathrm{Ac}, \mathrm{Ca}, \\
\mathrm{Ma}, \mathrm{Co}, \mathrm{Cu}, \\
\mathrm{Mu}\end{array}$ & $\begin{array}{l}\text { https:// } \\
\text { www.conchaytoro.com }\end{array}$ & 9.133 & Oui \\
\hline 2 & $\left|\begin{array}{ll}\text { Viña } & E l \\
\text { Principal } & \end{array}\right|$ & 1992 & Ma & https://www.elprincipal.cl & 90 & Oui \\
\hline 3 & $\begin{array}{l}\text { Viña } \\
\text { Aquitania }\end{array}$ & 1990 & Ma & http://aquitania.cl & 18 & Oui \\
\hline 4 & $\begin{array}{l}\text { Viña } \\
\text { Almaviva }\end{array}$ & 1997 & $\mathrm{Ma}$ & $\begin{array}{l}\text { http:// } \\
\text { www.almavivawinery.com }\end{array}$ & 60 & Oui \\
\hline 5 & $\begin{array}{l}\text { Viña } \\
\text { Undurraga* }\end{array}$ & 1885 & $\begin{array}{l}\text { Ma, Co, Le, } \\
\mathrm{Al}, \mathrm{Mu}\end{array}$ & $\begin{array}{l}\text { http://www.undurraga.cl/ } \\
\text { home }\end{array}$ & 1.350 & Oui \\
\hline 6 & $\begin{array}{l}\text { Viña Pérez } \\
\text { Cruz* }\end{array}$ & $\sim 1950$ & Ma & http://www.perezcruz.com & - & Oui \\
\hline 7 & $\begin{array}{ll}\text { Viña } & \text { Santa } \\
\text { Rita* }^{*} & \end{array}$ & 1880 & $\begin{array}{l}\text { Ca, Le, Ma, } \\
\text { Ra, Lo, }\end{array}$ & $\begin{array}{l}\text { http://www.santarita.com/ } \\
\text { chilehome }\end{array}$ & $>3.200$ & Oui \\
\hline 8 & $\begin{array}{l}\text { Viña De } \\
\text { Martino* }\end{array}$ & 1934 & $\begin{array}{l}\text { El, } \mathrm{Li}, \mathrm{Ch}, \mathrm{Ca}, \\
\mathrm{Ma}, \mathrm{Cc}, \mathrm{Mu}\end{array}$ & http://www.demartino.cl & - & Oui \\
\hline 9 & \begin{tabular}{|l} 
Viña \\
Tarapacá*
\end{tabular} & 1874 & Ma & http://www.tarapaca.cl & 2.600 & Oui \\
\hline 10 & Viña Odfjell* & 1990 & $\begin{array}{l}\text { Ma, Co, Lo, } \\
\text { Cq }\end{array}$ & http://odfjellvineyards.cl & 115 & Oui \\
\hline 11 & $\begin{array}{l}\text { Viña } \\
\text { Chocalán* }\end{array}$ & 1998 & $\mathrm{Ma}, \mathrm{Sa}$ & $\begin{array}{l}\text { https://chocalanwines.com/ } \\
\text { web/vinos }\end{array}$ & 340 & Oui \\
\hline 12 & $\begin{array}{l}\text { Viña Santa } \\
\text { Carolina }\end{array}$ & 1875 & $\begin{array}{ll}\mathrm{Ca}, \mathrm{Le}, \mathrm{Ma} \\
\mathrm{Cc}, \mathrm{Ra}, \mathrm{Co}, \\
\mathrm{Mu}\end{array}$ & http://www.santacarolina.cl & $>2.000$ & Oui \\
\hline 13 & $\begin{array}{l}\text { Viña } \\
\text { Cánepa* }\end{array}$ & 1930 & $\begin{array}{l}\mathrm{Li}, \mathrm{Ma}, \mathrm{Cc}, \\
\mathrm{Mu}\end{array}$ & http://canepawines.cl & - & Non \\
\hline 14 & $\begin{array}{l}\text { Viña } \\
\text { Carmen* }\end{array}$ & 1850 & $\begin{array}{l}\text { Ca, Ma, Le, } \\
\text { Ap, Co }\end{array}$ & http://www.carmen.com & - & Non \\
\hline 15 & $\begin{array}{l}\text { Viña Portal } \\
\text { del Alto }\end{array}$ & 1971 & $\mathrm{Ma}, \mathrm{Mu}$ & http://www.portaldelalto.cl & - & Non \\
\hline 16 & $\begin{array}{l}\text { Viña Santa } \\
\text { Ema* }\end{array}$ & 1956 & Ma, Le & http://santaema.cl & - & Non \\
\hline 17 & $\begin{array}{l}\text { Viña } \\
\text { MontGras - } \\
\text { Intriga* }\end{array}$ & 1993 & Ma, Le, Co & https://www.montgras.cl & 180 & Oui \\
\hline
\end{tabular}




\begin{tabular}{|c|c|c|c|c|c|c|}
\hline 18 & Viña Antiyal & 1996 & $\mathrm{Ma}$ & http://www.apaltagua.com & 21 & Oui \\
\hline 19 & $\begin{array}{l}\text { Viña } \\
\text { Aspaltagua }\end{array}$ & 1995 & $\begin{array}{l}\mathrm{Ma}, \mathrm{Sa}, \mathrm{Ap} \\
\mathrm{Co}, \mathrm{Cu}, \mathrm{Mu}\end{array}$ & http://www.apaltagua.com & 390 & Oui \\
\hline 20 & $\begin{array}{l}\text { Viña Cono } \\
\text { Sur* }\end{array}$ & 1992 & $\begin{array}{l}\mathrm{Li}, \mathrm{Ac}, \mathrm{Ca}, \\
\mathrm{Ma}, \mathrm{Sa}, \mathrm{Cc}, \\
\mathrm{Co}, \mathrm{Cu}, \mathrm{Mu}, \\
\mathrm{Bi}\end{array}$ & http://www.closandino.cl & - & Non \\
\hline 21 & $\begin{array}{l}\text { Viña } \\
\text { Emiliana* }\end{array}$ & $\sim 1999$ & $\begin{array}{l}\mathrm{Ca}, \mathrm{Ma}, \mathrm{Cc}, \\
\mathrm{Co}, \mathrm{Bi}\end{array}$ & http://www.emiliana.cl & 996 & Oui \\
\hline 22 & Viña Maipo* & 1948 & $\begin{array}{l}\mathrm{Ca}, \mathrm{Ma}, \mathrm{Ra}, \\
\mathrm{Mu}\end{array}$ & http://www.vinamaipo.com & - & Non \\
\hline 23 & $\begin{array}{l}\text { Viña } \\
\text { Morandé* }\end{array}$ & 1996 & $\mathrm{Ca}, \mathrm{Ma}$ & $\begin{array}{l}\text { http://www.morande.cl/ } \\
\text { web }\end{array}$ & - & Oui \\
\hline 24 & $\begin{array}{l}\text { Viña Tres } \\
\text { palacios }\end{array}$ & 1996 & $\mathrm{Ma}$ & $\begin{array}{l}\text { http:// } \\
\text { www.vinatrespalacios.cl }\end{array}$ & - & Non \\
\hline 25 & $\begin{array}{l}\text { Viña } \\
\text { Chadwick }\end{array}$ & 1942 & Ma & $\begin{array}{l}\text { http:// } \\
\text { www.vinedochadwick.cl }\end{array}$ & 25 & Non \\
\hline 26 & $\begin{array}{l}\text { Viña } \\
\text { Indómita }\end{array}$ & 2006 & $\mathrm{Ca}, \mathrm{Ma}, \mathrm{Bi}$ & http://www.indomita.cl & 1.400 & Oui \\
\hline
\end{tabular}

${ }^{1}$ : Vallées viticoles (du nord au sud) = El : Elqui ; Li : Limarí ; Ch : Choapa ; Ac :

Aconcagua ; Ca : Casablanca ; Le : Leyda ; Ma : Maipo ; Sa : San Antonio ; Cc : Cachapoal ; $\mathrm{Ra}$ : Rappel ; Ap : Apalta ; Co : Colchagua ; Al : Almahue ; Cu : Curicó ; Lo : Lontué ; Mu : Maule; $\mathrm{Cq}$ : Cauquenes; Bi : Biobío.

${ }^{2}$ : Avec activités œnotouristiques diverses (tours, salle de ventes, etc.) offertes dans le site internet du vignoble (certains vignobles non indiqués ici pouvant le faire à travers des tours opérateurs).

* : Vignobles avec le label du Code de développement durable de l'industrie du vin chilienne (http://www.sustentavid.org).

\section{NOTES}

1. Les textes de loi et autres documents cités ont été traduits par l'auteur de cet article à partir de leur version originale en espagnol.

2. Une évidente conséquence de l'héritage romantique à l'origine des premières mesures de conservation du début du XXe siècle, d'après Mata Olmo (2004).

3. Comme l'Association chilienne de professionnels du paysage (ACHIPPA) fondée en 1999 ou l'Initiative latino-américaine du paysage (LALI) née en 2012.

4. Comme la reconnaissance entre 2010 et 2012 de l'île de Chiloé en tant que site Sipam (Systèmes ingénieux du patrimoine agricole mondial) par la FAO - qui associe systèmes du patrimoine 
agricole et paysages. On peut également citer le concept de " Paysage de conservation ", mis en pratique dans trois communes du pays depuis 2008, et dont le but est de promouvoir la conservation et l'exploitation durable de la biodiversité dans des zones productives privées, à travers l'action conjointe avec le secteur public (ministère de l'Environnement).

5. La vallée du fleuve Maipo, par exemple, a accueilli des cépages français au milieu du XIXe siècle, qui sont à l'origine des grands vignobles actuels, avec des caves centenaires et des productions de grande qualité (« vins premiums ») (Del Pozo, 1999 ; Godoy \& Meza, 2014).

6. La surface des vignobles à vin a augmenté de 65202 ha en 1990 à 141918 ha en 2015 (celle des vignobles destinés à l'élaboration du pisco augmentant de 6506 à 8516 ha sur la même période ; chiffres de surfaces et production viticole, ODEPA, 2017a). Les vignobles à vin irrigués, eux, ont augmenté de 78028 à 121105 ha seulement entre 2000 et 2015 (chiffres du cadastre viticole national de 2015 et de 2000, ODEPA, 2017b).

7. Alors qu'en 2008, le Chili était le septième producteur et le cinquième exportateur de vin, principalement vers le Royaume-Uni et les États-Unis, les exportations de vin en bouteille ont augmenté de 57,8 \% entre 2005 et 2010 (données du Wine Institute dans Inalaf et al., 2012).

8. Le nombre de touristes entrés au Chili a doublé au cours des huit dernières années, passant de 2710024 en 2008 à 5640700 en 2016 (Sernatur, 2017), avec un taux de croissance annuel moyen de 12,09\% entre 2005 et 2015 (Infyde, 2016).

9. Promotion et organisation de fêtes des vendanges depuis 2001 par les mairies, Sernatur et les entreprises viticoles ; programme de promotion public «Bien Público Enoturismo Chile » lancé en 2013 par la Corporación de Fomento a la Producción (CORFO), Subsecertaría de Turismo, Sernatur, Vinos de Chile et Fedetur ; création de la Journée nationale du vin en 2015, promue par des associations de viticulteurs; première application mobile dédiée au tourisme du vin (CompassWine App), lancée en 2016 par Vinos de Chile et Sernatur, entre autres.

10. En Argentine, par exemple, où le développement de l'œnotourisme est également récent (il date des années 1990), les caves ont reçu 1205612 visiteurs en 2011 (voir Lignon-Darmaillac, 2014).

11. Dernières données officielles d'œnotourisme au Chili, issues du programme «Enoturismo Chile » élaboré par $+\mathrm{M}$ Consultores.

12. Augmentation interrompue en 2010 à cause du séisme au Chili qui a touché de nombreux domaines : 96 vignobles ouverts au tourisme en 2005, 102 en 2006, 64 en 2010, 73 en 2012, 78 en 2013 et 94 en 2016 (+M Consultores, 2014 ; Infyde, 2016).

13. Le passage dans la boutique est un élément essentiel des entreprises : $72 \%$ des touristes achètent au moins une bouteille lors de leur visite en 2012 (contre $62 \%$ en 2005). Certains vignobles à moindre production vendent entre 50 et $100 \%$ de celle-ci au sein même de leur établissement en augmentant le prix moyen par bouteille (+M Consultores, 2014 ; Infyde, 2016).

14. Ce qui représente $28 \%$ des 339 vignobles enregistrés dans le pays possédant une cave à vinification - et $16 \%$ si l'on considère le total de plus de 600 vignobles du pays sans cave de vinification mais autorisés à produire et à exporter du vin. Une grande partie d'entre eux développe aussi une certaine activité œnotouristique de manière non officielle (Infyde, 2016).

15. En 2015, $50 \%$ des 41 vignobles interrogés déclarent que l'œnotourisme représente entre 1 et $5 \%$ de leurs revenus pour cette année-là et $19 \%$ le situent entre 6 et $10 \%$; et parmi 43 vignobles interrogés, 56 \% déclarent une bonne ou très bonne évolution des revenus de cette activité dans les cinq dernières années (Infyde, 2016).

16. Lors du séminaire « Du vin, de la gastronomie et de la ruralité » à la Bibliothèque nationale du Chili (Aravena, 2015).

17. Voir le prologue de Mario Fregoni - président d'honneur de l'Organisation internationale de la vigne et du vin (OIV) -, dans l'ouvrage collectif Patrimonio vitivinícola, Aravena (éditeur), 2015.

18. Ainsi, le cépage carménère, que l'on croyait disparu avec la crise de phylloxera de la fin du XIXe siècle en Europe, a été redécouvert dans la zone centrale du Chili dans les années 1990 - 